\title{
Study on diesel engine exhaust particle separation based on gas-solid two-phase flow theory
}

\author{
Xiaopeng Xie ${ }^{1} \cdot$ Heng Huang ${ }^{2} \cdot$ Xindan Zhang $^{2} \cdot$ Yaowu Shen ${ }^{3} \cdot$ Youpeng Chen ${ }^{3}$
}

Received: 30 January 2019 / Accepted: 24 January 2020 / Published online: 13 February 2020

(c) Springer Nature Switzerland AG 2020

\begin{abstract}
Particulate matter in diesel exhaust is one of the primary sources of environmental pollution. This paper mainly studies the purification treatment of particulate matter in exhaust gas. It also studies the stress conditions and moving process of particulates in straight and elbow pipes respectively and explores their capture efficiency on the basis of that the gassolid two-phase flow composed of particulates and gas is a basic fluid state. This paper mainly analyzes the influence of straight and elbow pipe on the moving process of particulates. It also discusses the impact of the structure parameters of the elbow on the exhaust pressure of inlet and outlet of pipes to provide basis for its designing. In addition, experiment is carried out to verify the capture efficiency of particulates by elbow pipes under different working conditions of diesel engines. The experiment results show that the capture efficiency is improved and separation of particulates is more effective.
\end{abstract}

Keywords Diesel engine $\cdot$ Particulate matter - Gas-solid two-phase flow · Elbow pipe · Separation

\section{Abbreviations}

$\rho_{g} \quad$ Density of exhaust gas

$u_{g} \quad$ Vertical speed of exhaust gas

$v_{p} \quad$ Horizontal speed of particulates

$d_{p}$ Diameter of particulates

$D$ Pipe diameter

$F_{D} \quad$ Vertical upward resistance force

$u_{s} \quad$ Centrifugation velocity

$v_{g} \quad$ Horizontal speed of exhaust gas

$\rho_{p} \quad$ Density of particulates

$u_{p} \quad$ Vertical speed of particulates

$\mu$ Kinematic viscosity

$F_{g} \quad$ Gravity force

$u_{r} \quad$ Centripetal velocity

\section{Introduction}

Diesel engine is widely used among large machines for its high power [8]. However, for its rough working mode, fuel can hardly burn efficiently. As a result, there are many harmful substances contained in the exhaust gas, among which nitrogen oxide and particulates are the most prominent, contributing to a serious impact on the environment. Especially those particulates with soot in the majority not only cause serious environment problems, but also do harm to human health $[1,7,15]$. Therefore, it is of vital importance to collect and purify particulates in the exhaust gas.

At present, the main way to deal with particulates in the diesel exhaust is filtering. One of the common methods is to add a DPF particle separator in the exhaust pipe. By studying the size distribution of particulates under different working conditions, Ji et al. [5] and Morawska et al. [9]

$\triangle$ Heng Huang, huangheng0921@126.com; Xiaopeng Xie, xiexp@scut.edu.cn; Xindan Zhang, 648594036@qq.com; Yaowu Shen, 306908510@qq.com; Youpeng Chen, 814744475@qq.com | ${ }^{1}$ City College of Dongguan University of Technology, Dongguan 523419, People's Republic of China. ${ }^{2}$ South China University of Technology, Guangzhou 510640, People's Republic of China. ${ }^{3}$ Guangzhou Nanyang Polytechnic College, Guangzhou 510900, People's Republic of China. 
found that the size of particulates was related to the rotating speed and the load of the diesel engine. Besides, different fuel additives also have an effect on the formation of particulates. Barrios et al. [2] investigated the effects of oxygenated compounds as fuel additives on the exhaust emission, which performed better as the oxygen concentration increased. Yi [12] defined the distribution of particulates in the catcher by establishing a three-dimensional macro numerical model. In addition, Choi et al. [3] studied the influence of different separator's porosities on pressure drop, capture and regeneration efficiency. Zuccaro et al. [16] established the corresponding mathematical model to consider the air flow field and deposition characteristics of particulates inside the DPF. Tsuneyoshi and Yamamoto [10] adopted a new filter unit, which was improved from the traditional square filter grid, with a higher capture efficiency and lower pressure drop.

This paper mainly separates particulates by changing their moving track, avoiding the pressure effect on the exhaust process due to filtration from small holes. Previous studies pointed out that the particulates and gas in the exhaust form a gas-solid two-phase state [6,13], which provides the thought to separate these particulates in the point of hydromechanics. This study investigates the moving process of particulates, considered as discrete phase in the pipes, based on the gas-solid two-phase theory. A particle separation method based on gas-solid two-phase flow theory is put forward, as well as the moving process mathematical model of particulates in the pipe. Based on the previous work and research, techniques and special design are applied to pipe wall, so that particulates are adsorbed and separated by collision with the pipe wall due to the changing track.

\section{Mathematical model of particulates}

First of all, the surface morphology is firstly observed by electron microscope to understand the shape of particulates in the exhaust. In the experiment, particulate matters are observed by the magnification of $200,1.00 \mathrm{~K}, 5.00 \mathrm{~K}$ and $20.00 \mathrm{~K}$ times respectively, and the result is shown in Fig. 1.

As seen in the figure, the particle size in the exhaust is quite small, with the diameter ranging from 0.1 to $10 \mu \mathrm{m}$. Individual particulate is mostly spherical or spheroidal, while multiple particulates are aggregated together in the form of block or chain. This paper mainly analyzes the moving process of a single particle in the pipeline. To simplify the calculation, the shape of the particulates in the exhaust is assumed spherical. The moving process is simulated using the corresponding mathematical model, with force analysis of spherical particulates in straight and elbow pipes, respectively. Theoretical analysis about capture effect on particulates is conducted based on the comparison of moving process in straight and elbow pipes.

Assume that the density, horizontal speed, vertical speed of exhaust gas in the pipe from diesel engine are $\rho_{g}, v_{g}, u_{g}$ respectively. While the density, horizontal speed, vertical speed and diameter of particulates are $\rho_{p}, v_{p^{\prime}}, u_{p^{\prime}}, d_{p^{\prime}}$ respectively. The kinematic viscosity is $\mu$ and the pipe diameter is $D$. In this paper, Isuzu 4JB1 diesel engine is used. According to corresponding working conditions, when the temperature is $300^{\circ} \mathrm{C}$, the above parameters are, $\rho_{g}=0.615 \mathrm{~kg} / \mathrm{m}^{3}, \rho_{p}=750 \mathrm{~kg} / \mathrm{m}^{3}, \nu_{g}=30 \mathrm{~m} / \mathrm{s}, \mu=2.96$ $\times 10^{-5} \mathrm{~Pa} \cdot \mathrm{s}$. Suppose that the diameter of particulate is $1 \mu \mathrm{m}$, and the pipe diameter is $50 \mathrm{~mm}$. Substitute the values of relevant parameters to the Reynolds Equation:

$\operatorname{Re}=\frac{\rho v d}{\mu}$

The Reynolds number is: $\operatorname{Re}=0.012\left|v_{g}-v_{p}\right|<1$. Therefore, the resistance subjecting to the particulates is in the Stokes area [14], and the resistance coefficient is:

$C_{D}=\frac{24}{\operatorname{Re}}$

\subsection{Settling moving of particulates in straight pipe}

To begin with, this paper studies the moving situation of particulates in straight pipe. In the pipeline, we analyze the stress situation of particulates as they interact with gas. As discussed above, the shape of particulates is assumed to be spheroid, and we ignore temperature changes in the pipeline. As $\rho_{g} \ll \rho_{p}$, the buoyancy of particulates is negligible. As a result, particulates are mainly constrained by gravity and movement resistance during moving process. As shown in Fig. 2, since particulates enter the pipeline at the same horizontal speed of gas, no resistance force takes effect in the horizontal direction. And in the vertical direction, particulates are settled by gravity force $F_{g}$. Meanwhile, a vertical upward resistance force $F_{D}$ is applied by gas, the direction of which is opposite to the relative speed. Resistance force thus can be written as follows:

$F_{g}=m g=\frac{1}{6} \pi d_{p}^{3} \rho_{p} g$

$F_{D}=\frac{1}{2} C_{D} \pi r_{p}^{2} \rho\left(u_{g}-u_{p}\right)^{2}=\frac{1}{8} C_{D} \pi d_{p}^{2} \rho\left(u_{g}-u_{p}\right)^{2}$

The moving process of particulates in the pipeline is in accordance with Newton's law: $\sum F=m a$, which can be also described as: 


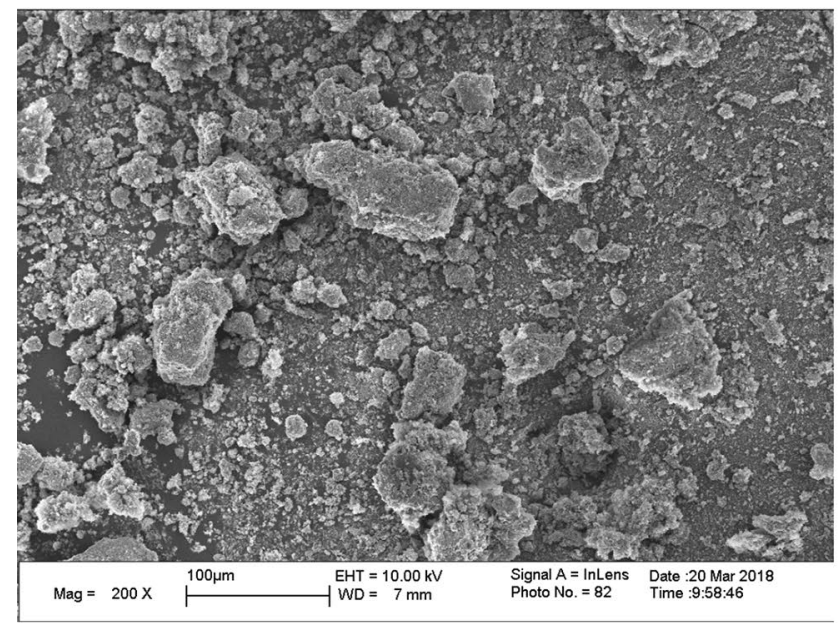

(a) $200 \mathrm{X}$

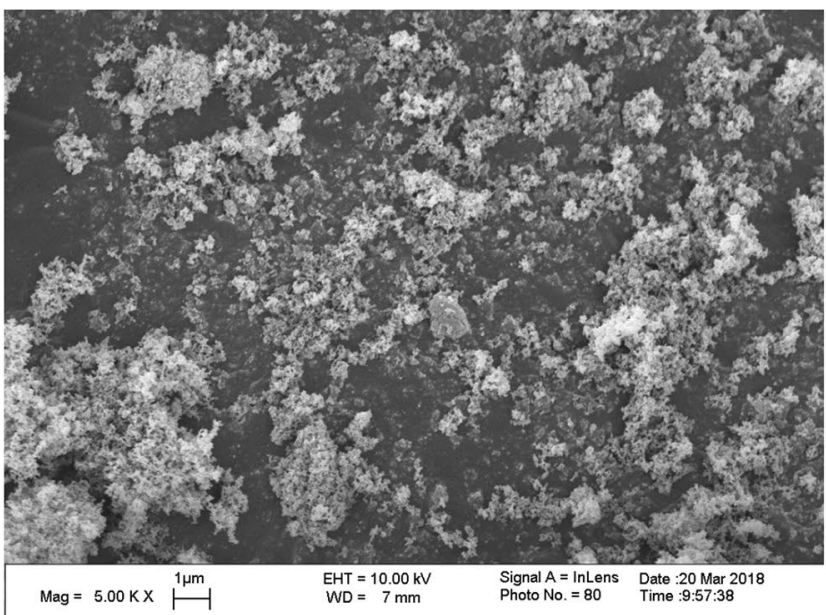

(c) $5.00 \mathrm{~K} \mathrm{X}$

Fig. 1 Electron micrograph of particulates. This figure tells the surface morphology of particulate matters. The magnification is 200 , $1.00 \mathrm{~K}, 5.00 \mathrm{~K}$ and $20.00 \mathrm{~K}$ times respectively. It aims to describe the

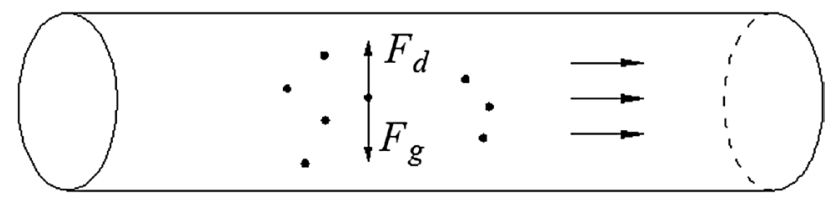

Fig. 2 Force situation of particulates. This figure describes the force the particles subject to in the pipeline. They are gravity force $F_{g}$ and vertical upward resistance force $F_{D}$. It helps to analyze the motion process of particulate matters

$$
F_{g}-F_{D}=m a
$$

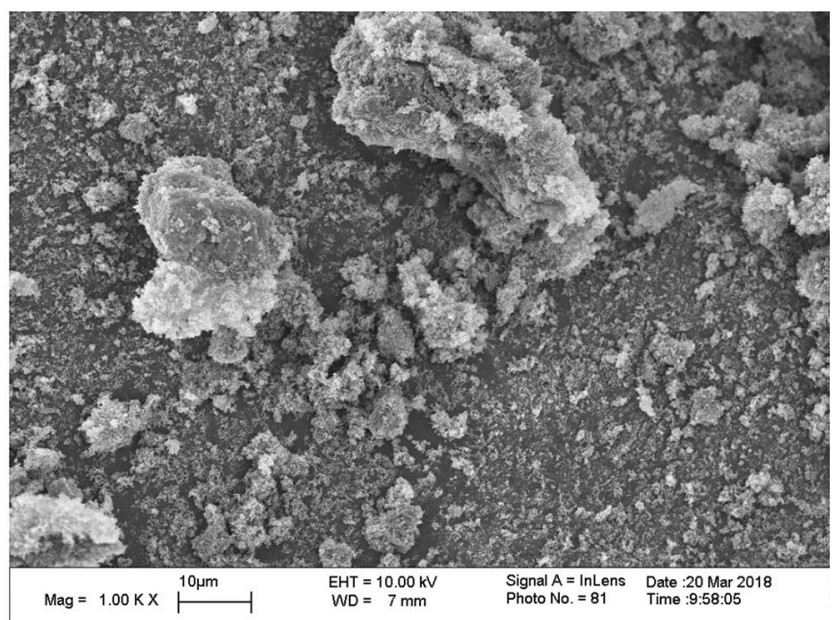

(b) $1.00 \mathrm{~K} \mathrm{X}$

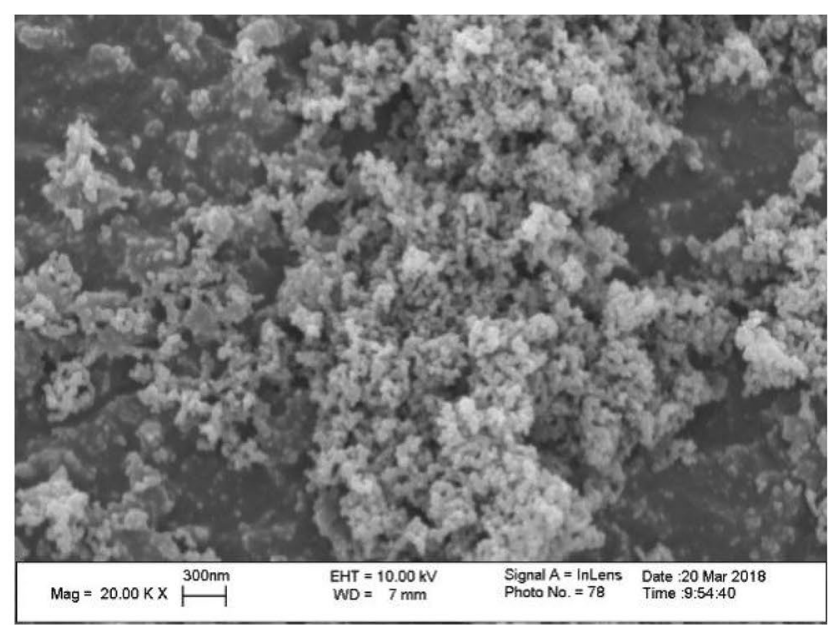

(d) $20.00 \mathrm{~K} \mathrm{X}$

size and surface of particles to understand the formation process of particulate matters better

Substitute Eqs. (1)-(4) into Eq. (5), and the Equation can be simplified as:

$\frac{d u_{p}}{d t}=\frac{u_{g}-u_{p}}{\frac{\rho_{p} d_{p}^{2}}{18 \mu}}+g$

As shown in Eq. (5), gravity force and movement resistance force affect the movement of particulates in the vertical direction. As the speed and stress change and the acceleration falls to 0 , namely $\frac{d u_{p}}{d t}=0$, the value of resistance force in the vertical direction is equal to that of gravity force for particulates, and the vertical velocity of particulates stays stable. In this case, settling velocity of particulates is inferred as: 
$u_{p t}=\frac{\rho_{p} d_{p}^{2} g}{18 \mu}$

As indicated by Eq. (7), particulates settle down at the speed of $u_{p t}$ in the vertical direction of straight pipe. Assume that particulates settle from the top to the bottom of the pipe at the speed of $u_{p t}$, the horizontal distance required for settlement, namely length of pipe, can be calculated as:

$I_{p t}=\frac{18 \mu v_{p}}{\rho_{p} d_{p}^{2} g} D=\frac{D v_{p}}{\frac{\rho_{p} d_{p}^{2} g}{18 \mu}}$

\subsection{Settling moving of particulates in elbow pipe}

Same as in the straight pipe, particulates are also subject to gravity force and resistance force in the elbow pipe during moving process. However, differ from straight pipes in which the vertically downward velocity $u_{g}$ is caused merely by gravity, the vertical velocity of particulates in the elbow pipe also includes centripetal velocity $u_{r}$ and centrifugation velocity $u_{s}$ due to centrifugal moving. The direction of $u_{s}$ is parallel to the pipe, while $u_{r}$ is perpendicular to the pipe and points to the center of the trajectory. The moving process of particulates in elbow pipes can be described as in Fig. 3.

The velocity $u_{g}$ generated by gravity can be decomposed into the directions of $u_{r}$ and $u_{s}$. The component of $u_{s}$ will not collide with the pipe wall. However, the combination of $u_{g}$ and $u_{r}$ will accelerate the collision process. Consequently, the force is decomposed in the vertical direction, and according to Eq. (5), the moving process can be expressed as follows:

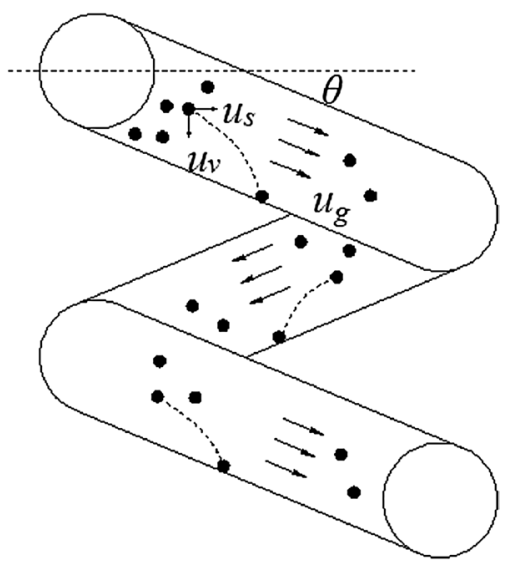

Fig. 3 Schematic diagram of particulates moving. This figure illustrates the force situation of particles in the elbow. The force decomposition is made in all directions $-\frac{1}{8} C_{D} \pi d_{p}^{2} \rho_{g}\left(u_{p}-u_{g} \sin \theta\right)+\frac{1}{6} \pi d_{p}^{2} \rho_{p} g=m \frac{d u_{p}}{d t}$

When the force in the vertical direction approaches 0 , the moving of particulates becomes stable, namely $\frac{d u_{p}}{d t}=0$. In this case, the final velocity of particulates in steady state can be calculated as follows:

$u_{p s}=u_{g} \sin \theta+\frac{\rho_{p} d_{p}^{2} g}{18 \mu}$

As described in Eq. (10), the final vertical velocity of particulates is $u_{p s}$. Similarly, assume that particulates settle from the top to the bottom of the pipe at the speed of $u_{p s^{\prime}}$ the horizontal distance required for settlement, namely length of pipe, can be calculated as:

$I_{p s}=\frac{D v_{p}}{u_{g} \sin \theta+\frac{\rho_{p} d_{p}^{2} g}{18 \mu}}$

Further, substitute the corresponding values into each parameter, the terms in Eq. (11) can be calculated as follows:

$$
\frac{\rho_{\rho} d_{p}^{2} g}{18 \mu}=1.41 \times 10^{-5} \text {, the value of } u_{g} \text { is } 30 \mathrm{~m} / \mathrm{s} . \theta \text { is related }
$$

to the inclination of pipes and it is at least $10^{\circ}$. In this case, $u_{g} \sin \theta \gg \frac{\rho_{p} d_{p}^{2} g}{18 \mu}$.

Based on the above analyses, the length required for settlement in straight and elbow pipes can be compared as:

$I_{p t} \gg I_{p s}$

As shown in Eq. (12), particulates settle down because of gravity in straight pipes, while the settlement is accelerated in elbow pipes due to centrifugal movement. In elbow pipes, the faster the trajectory of particulates changes and the shorter the distance is needed. In conclusion, the capture efficiency for particulates in elbow pipes is much higher than that in straight pipes. Consequently, the purification of particulates is much better in elbow pipes.

\section{Simulation analysis of particulates moving process}

In this section, numerical calculation of particulates moving process respectively in straight and elbow pipes are carried out based on ANSYS. Besides, sensitivity simulations are performed to probe the impacts of structural parameters of elbow pipes on the capture efficiency and working efficiency of diesel engine. 


\subsection{Simulation analysis of particulates in straight and elbow pipes}

The capture efficiencies of particulates in straight and elbow pipes are compared by ANSYS simulation results. Their physical models are as shown in Fig. 4. The diameters of both straight and elbow pipes are $50 \mathrm{~mm}$, the length of straight pipe is $400 \mathrm{~mm}$, and the curved pitch and helix angle of elbow pipes is $100 \mathrm{~mm}$, and $540^{\circ}$.

Given that the flow field in the pipe is steady and isothermal, the fluid control equation and RNG $k-\varepsilon$ turbulence model equations were used in the simulation process $[4,11]$. After setting the corresponding boundary conditions mentioned above to physical models, the trajectory and number of particulates in the inlet and outlet of the pipe are simulated to obtain the moving process of particulates in pipelines and to compare the collection efficiency by straight and elbow pipes. Assume that the size of particulates distributed evenly from 0.1 to $2 \mu \mathrm{m}$, and the fluid medium is air. Particulates are evenly distributed at the inlet of pipe, entering at a speed of $30 \mathrm{~m} / \mathrm{s}$. Earlier research have found out an absorbent material similar to the sponge, wet by water, arranged on the inner wall of pipe, which will adsorb most of particulates in colliding with it. As a result, it is defined in this paper that particulates are captured when collision with wall for simplifying the simulation. While particulates escape from pipeline are considered uncaptured during the moving process.

The simulated trajectories of particulates in straight and elbow pipe are shown in Fig. 5, and the simulated particle number captured in the pipeline are shown in Fig. 6.

As shown in Fig. 5, for better visualization, 50 particulates of different diameters are taken to record trajectories. Clearly, particulates move smoothly in a laminar flow state. Particulates settle down under the influence of gravity force and movement resistance force. The trajectories in the straight pipe are much more steady when compared with to those in the elbow pipe, and particulates tend to settle gradually in the flow process. While in elbow pipe, particulates move centrifugally leading to a shift in the track due to the centrifugally force. Settling speed tends to be relatively higher under the influence of gravity

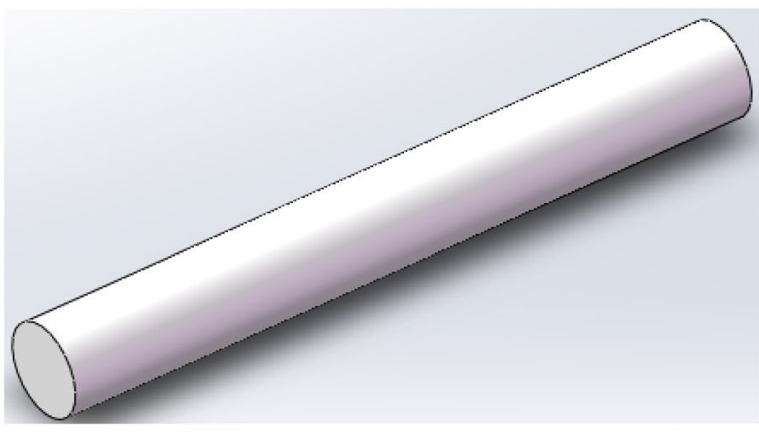

(a) Straight pipe

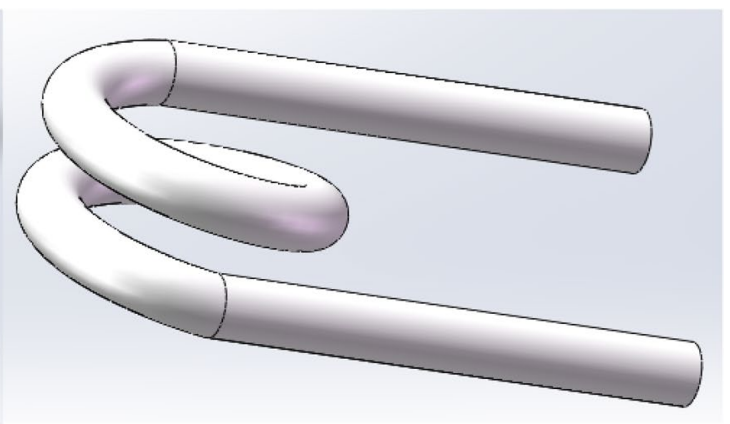

(b) Elbow pipe

Fig. 4 Physical model of pipelines. This figure depicts the appearance of the straight and elbow pipe. It tells reader how the pipe is and which kind of device particles move in

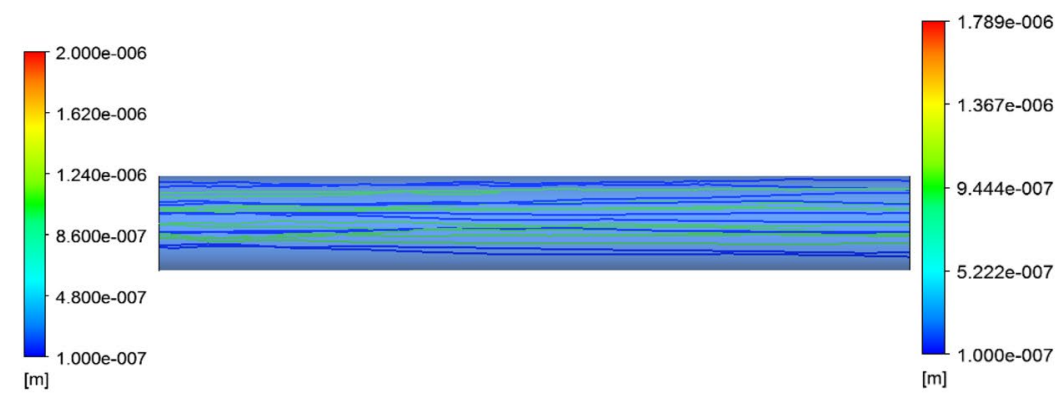

(a) Straight pipe

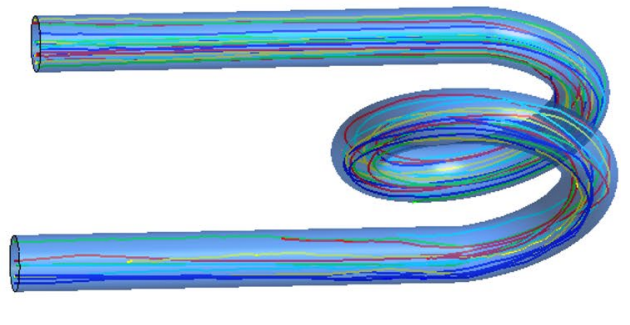

(b) Elbow pipe
Fig. 5 Particulates moving trajectories. This figure describes the trajectories of particulate matters in straight and elbow pipes. It tells how particles move in the pipe by means of simulation. This figure shows that it is more conducive to the sedimentation of the particles in elbow pipes than in straight pipes 


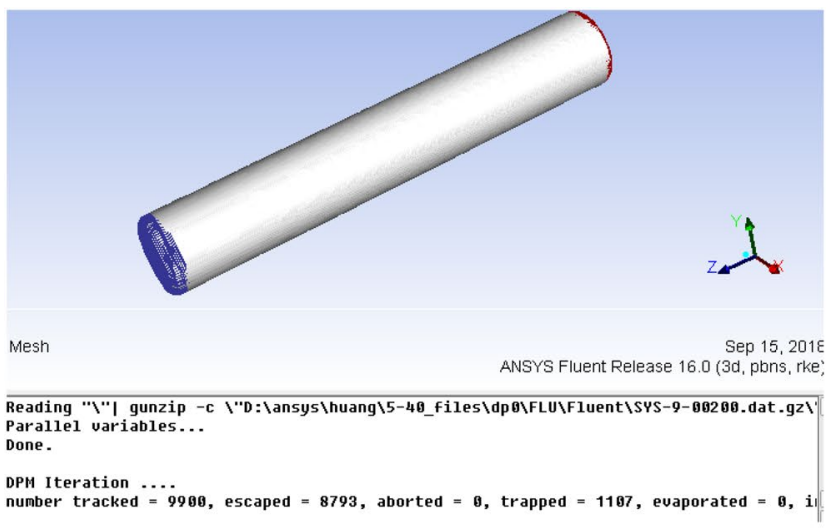

(a) Straight pipe

Fig. 6 Particulates number. This figure shows the number of particles at the inlet and outlet in straight and elbow pipes. It illustrates how much the number of particles captured by straight and elbow

force, resistance force, etc., which leads to a more trending settlement.

Corresponding to Fig. 5, escape and capture of particulates in straight and elbow pipes are shown in Fig. 6. As supposed that particulates are captured once they collision with pipe wall, settlement distance can reflect the capture efficiency. As shown in Fig. 6a, the capture efficiency is $11.18 \%$ in the straight pipe, with 9900 particulates in the inlet while 8793 in the outlet. In other words, only 1107 particulates are captured. While in Fig. 6b, the capture efficiency is much higher $(56.45 \%)$ in the elbow pipe. By contrast, there are 10,360 particulates in the inlet, while 4515 in the outlet, which implies that 5845 particulates are captured.

As demonstrated in Figs. 5 and 6, both straight and elbow pipes have a certain effect on particulates collection. The simulation results show that trajectory can change more easily in elbow pipes with a higher possibility to collide with walls. As mentioned above, in the designed device, materials are applied to the wall so that most particulates are adsorbed and captured once a collision occurs, which makes elbow pipes effective to purify the exhaust of diesel engine. However, pressure drop is a common problem for elbow pipes, resulting in a reduction in engine efficiency. Therefore, main structural parameters of elbow pipes need to be taken into consideration to simulate the effect of exhaust pressure at the inlet and outlet of elbow pipes.

\subsection{Optimization design of structural parameters for elbow pipes}

As shown in the theory calculation results and simulation analyses above, elbow pipes have a better effect on

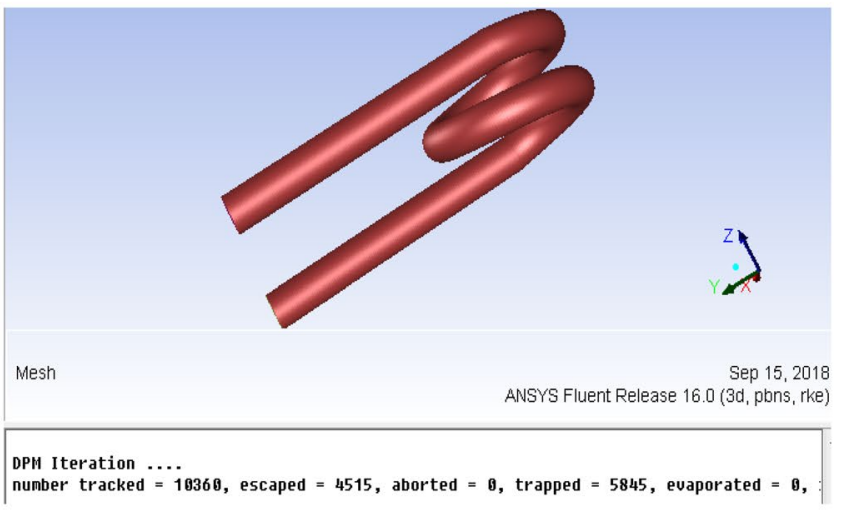

(b) Elbow pipe

pipes. Meanwhile, it also depicts the elbow pipe is more conductive to the capture of particles than straight pipe

exhaust purification compared with straight pipes. But exhaust flow will encounter some resistance due to structural features of elbow pipes, resulting the pressure drop and lower engine efficiency. To explore these influencing factors, the main structural parameters, like pipe diameter, curved pitch and helix angle, will be discussed in this section. Considering the design with the working condition, the pipe diameter of the exhaust is almost $50-60 \mathrm{~mm}$, values of each set of structure parameter are given in Table 1.

Assume that the particulate diameter is $1 \mu \mathrm{m}$ in the simulation. The influences of pipe diameter, curved pitch and helix angle on the pressure drop are shown in Fig. 7.

As shown in Fig. 7a, obviously, the size of pipe diameter plays a positive role in pressure drop. The smaller the pipe diameter, the greater the pressure $\operatorname{drop}(\Delta \mathrm{P})$, and the more obvious impact on the efficiency of engine. This implies that pipes with large diameter is beneficial to reduce the pressure drop and conducive to the flow of gas. Compared with pipe diameter, helix angle has less influence on pressure drop (Fig. 7b). The lager the helix angle, the longer distance the exhaust needs during flow

Table 1 Structure parameters of elbow pipe

\begin{tabular}{lll}
\hline Pipe diameter D $(\mathrm{mm})$ & Pitch L $(\mathrm{mm})$ & $\begin{array}{l}\text { Spiral } \\
\text { angle } \\
\phi\left(^{\circ}\right.\end{array}$ \\
\hline 50 & 100 & 360 \\
60 & 110 & 450 \\
70 & 120 & 540 \\
80 & 130 & 630 \\
90 & 140 & 720 \\
100 & 150 & 810 \\
\hline
\end{tabular}




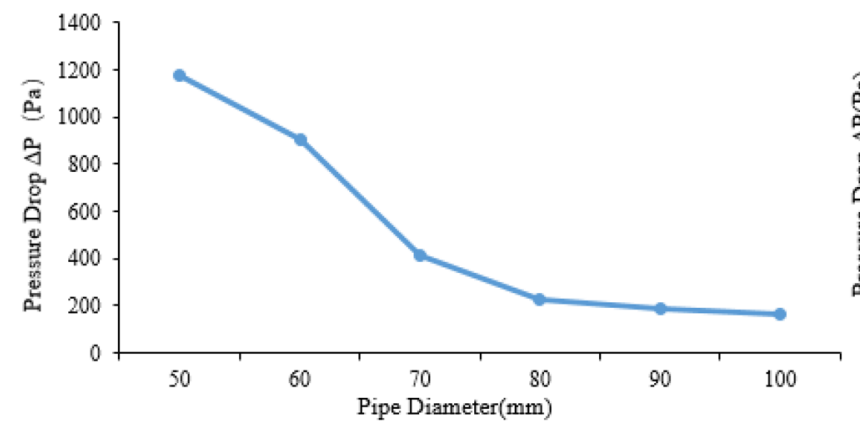

(a) Effect of pipe diameter

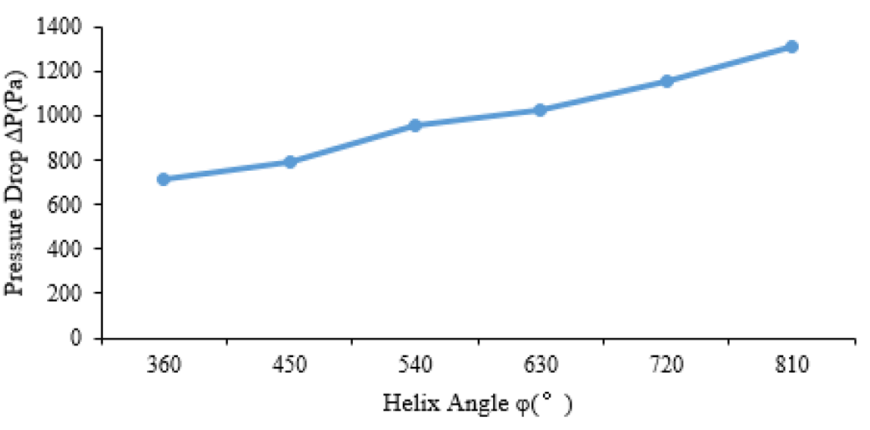

(b) Effect of helix angle

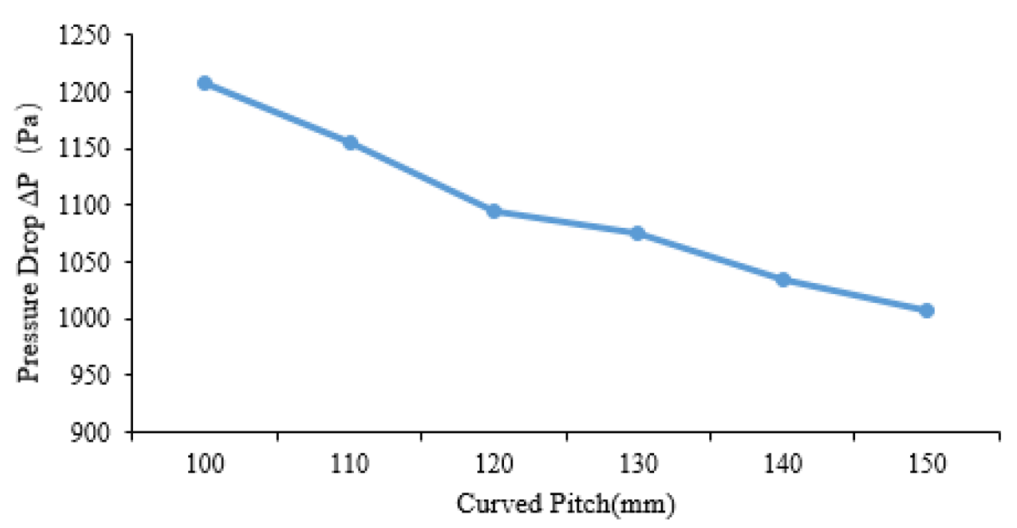

(c) Effect of curved pitch

Fig. 7 Effect of structure parameters on pressure drop. This figure shows the effect of different structural parameters of elbow pipe on the pressure difference at the inlet and outlet of the exhaust

process. More resistance need to be overcome due to original track changed, which makes pressure drop greater. Besides, changes of curved pitch have the least influence on the pressure drop. As we can see in Fig. 7c, pressure drop decreases as the curved pitch increases, which indicates that the space between two bends is conducive to the gas flow.

In conclusion, for elbow pipes collecting particulates, the pipe diameter, curved pitch and helix angle all affect the pressure drop and therefore the working efficiency of engine. It can be seen from Fig. 7 that pipe diameter has the greatest effect on the pressure drop of the exhaust among the three factors, while the curved pitch has the least. Consequently, these three factors should be taken into consideration when designing the purification device.

\section{Experiment and analysis}

Based on the aforementioned analyses, an elbow-pipe exhaust purification device is designed. In this section, we introduce the device and verify its effect on particulates pipe. It tells effect of pipe diameter, helix angle and curved pitch on the pressure drop, which means what to consider

collection. The schematic structure of the elbow-pipe exhaust purification device is shown in Fig. 8. It consists of eight major parts mentioned in the figure.

The experiment is designed to explore the purification effect of "elbow-pipe" device on particulates collection. Test bed consisting of three parts including diesel engine, purification device and detection device is shown in Fig. 9. As diesel engine works, the exhaust flows into the pipe and particulates are separated and collected through purification device. The purification effect is examined by the detection device.

To discuss the capture efficiency of the device under different working conditions, three working conditions of diesel engine, i.e. starting, accelerating and running smoothly, are tested in the experiment. Type NHT-6 Opaque Smoke Meter is used as detection device to examine the original and using purification device content of particulates in the exhaust under the three working conditions respectively. NHT-6 opaque smoke meter is a commonly used device to detect exhaust gas components and their concentrations in the experiment. Its principle is to detect the concentration of particulates in the fluid by 


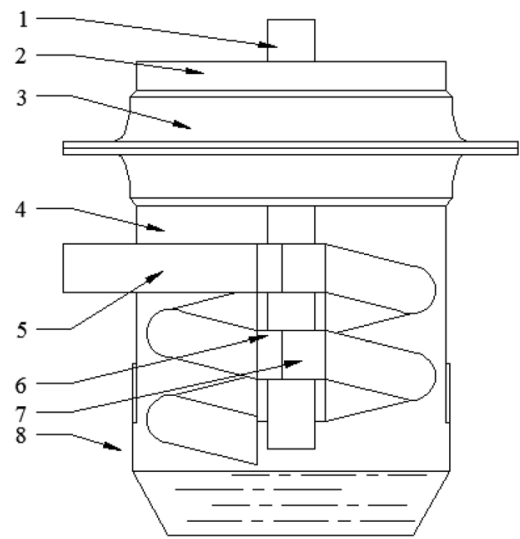

1-Exhaust pipe; 2-Sealing cap; 3-Flange; 4-Cylinder; 5-Elbow;

6-Pipe wall; 7-Pipe joint; 8-Liquid

Fig. 8 The structure schematic of the device. This figure shows the design and components of the device. It tells every part the purification device is mainly composed of, which makes it easier for readers to understand the principle of the purification device

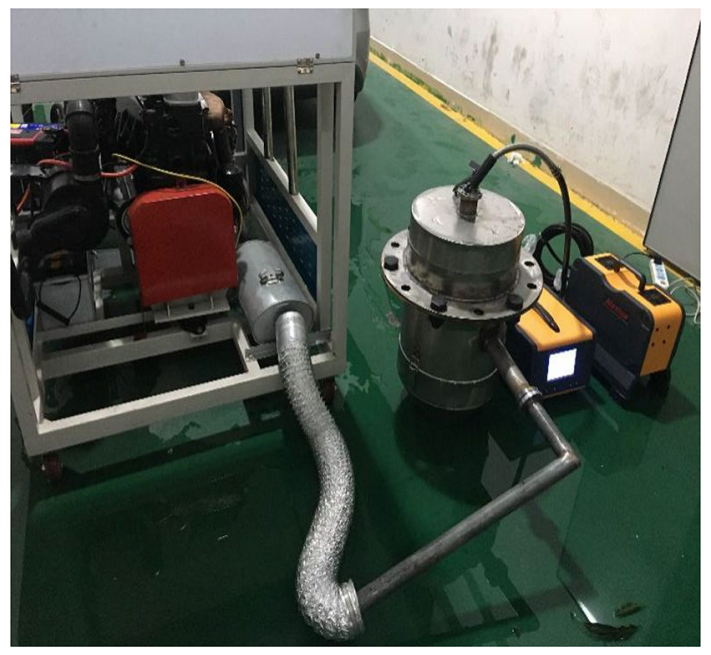

Fig. 9 Experiment bed. It is the experiment bed used in the experimental part of this paper. It shows various parts contained in the gantry. It can be seen from the figure how the experiment is carried out. It includes the exhaust gas generation part, purification part and detection part

"shading method". The test results are expressed by opacity (\%), which reflects the concentration of particulates in the device, and the resolution is $0.1 \%$. The larger the opacity value is, the greater the concentration of particulates is. In the experiment, data is collected every $10 \mathrm{~s}$ at each stage. The results are listed in Fig. 10.

Figure 10a shows that the smoke value is relatively high at starting stage. At that time, the temperature in the combustion chamber is low because diesel has just been compressed and ignited, so that fuel cannot be completely burned. More resistance should be overcome for piston movement. As a result, more particulates are contained in the exhaust. Compared with Fig. 10a, b shows that the smoke value is also high when engine is accelerating. At this stage, with the sudden increasing fuel injected into the combustion chamber, the concentration of particulates increases due to incompletely burned fuel. When the diesel engine is in stable condition (Fig. 10c), the fuel injected into the combustion chamber can be burned fully. The injection and compression of fuel are in balance. Particulates contained in the exhaust are much less than that in starting and acceleration stage.

Particulates in exhaust can be separated effectively with designed "elbow-pipe" exhaust purification device. According to the experiment and figures shown above, at the starting stage, the capture efficiency can be reached almost $60 \%$, while at the accelerating stage, the efficiency is lower with the same opacity. When engine runs smoothly, the opacity is much lower and can be reached nearly $20 \%$. The purification device has obvious effects on emission reduction under three working conditions for diesel engine. Compared to the starting and accelerating stages, the device can reduce the particulate concentration to a lower level when engine runs smoothly, and the capture efficiency can reach as high as $60 \%$. The experiment results suggest that the designed device in this paper can help to collect particulates in exhaust well and keep the emission at a lower level.

\section{Results and discussion}

In this paper, theoretical calculation, modeling, simulation and test experiment are carried out to study the moving process of exhaust, especially trajectories of particulates, in the pipeline. Capture efficiencies of straight and elbow pipes are compared. And the influence of pipe structure parameters on pressure drop has been discussed. Major conclusions are summarized as follows:

(1) Particulates settle down in pipelines due to gravity, movement resistance, etc. According to theoretical calculations, elbow pipes can have a larger impact on particulate trajectories and have a higher capture efficiency compared to straight pipes.

(2) The structure of elbow pipe has an impact on the emission pressure of inlet and outlet of pipes, causing pressure loss and low engine efficiency. The effect of different structure parameters on pressure drop varies. Pressure drop decreases as pipe diameter and curved pitch increase, while it increases with helix angle. Moreover, compared with pipe diameter and

\section{SN Applied Sciences}




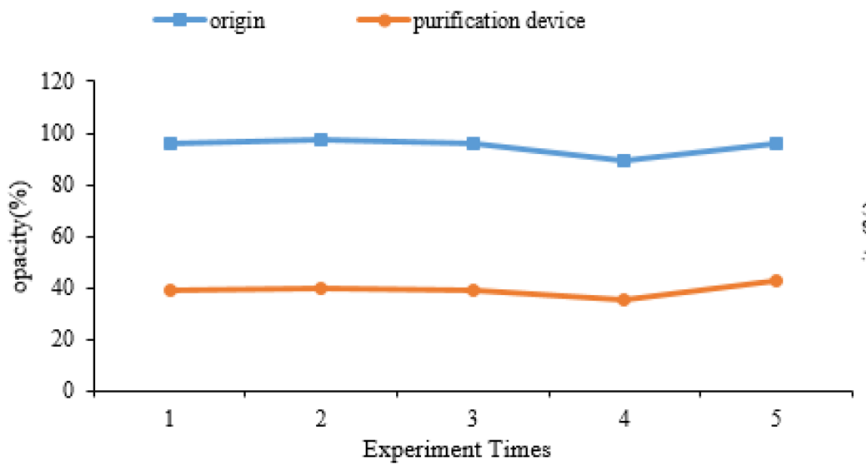

(a) Starting

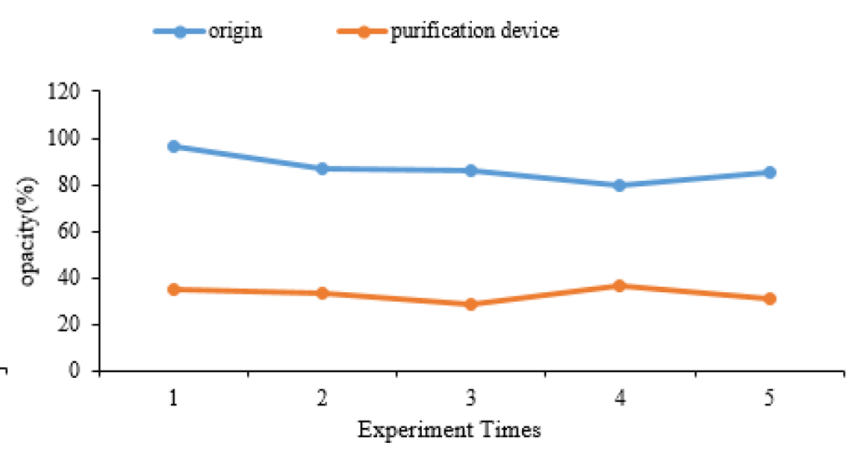

(b) Accelerating

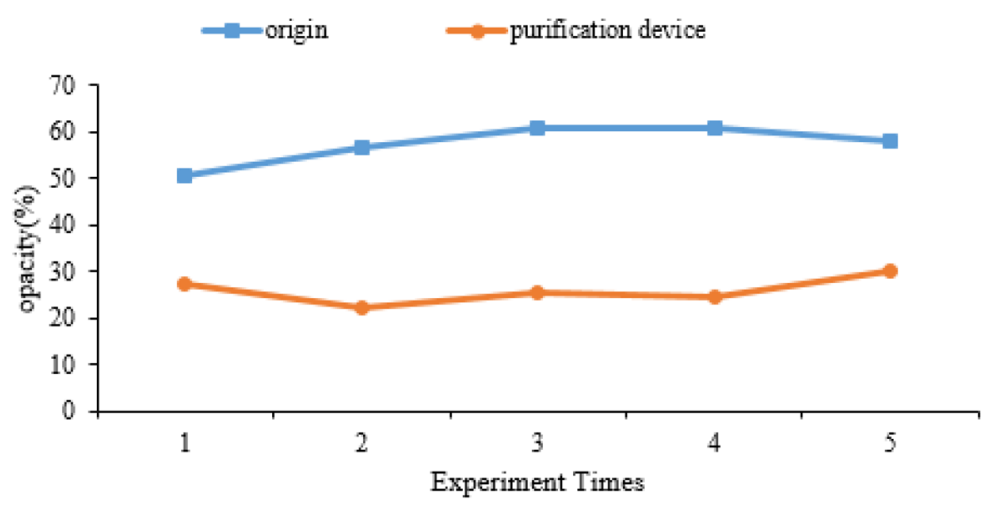

(c) Running smoothly

Fig. 10 Capture efficiency under different working conditions. This figure shows the capture efficiency of the purification device under three different working conditions. Under every single condition,

helix angle, curved pitch has little effect on pressure drop.

(3) The number of particulates contained in the exhaust varies under different working conditions of diesel engine. The designed "elbow-pipe" device has a more significant effect on particulates collection, and can effectively reduce particulate emission in the exhaust.

\section{Conclusions}

This paper mainly discusses a new way to purify particulate matters in the exhaust of the diesel engine. The gas-solid two-phase theory is applied to analyze the motion of gas and particulates respectively. Since the force and motion process of particulate matters are carried out individually, it is possible to separate the particles by applying other forces through the device. Based on the method, a new way to purify the exhaust is applied to design the device. The advantages and disadvantages are taken into considerations compared with the DPF. By the way, it's a new way to analyze the exhaust and separate the particulates. the comparation of the capture efficiency with purification device and the original is made

Acknowledgements The authors gratefully acknowledge the support for this research from Guangzhou Science Technology and Innovation Commission (Grant No. 201707010151) and Dongguan Science Technology Commission (Grant No. 20185071511637).

Author's contributions In this paper, XX carried out the whole idea about the project, participated in the building of the mathematic model. $\mathrm{HH}$ analyzed the motion process of gas and particulates, designed the purification device and drafted the manuscript. XZ helped with the mathematic model. YS participated in the drawing of the purification device. YC performed the calculation of gas and particulate matters. All authors read and approved the final manuscript.

Funding The funding of this paper is from the Guangzhou Science Technology and Innovation Commission (Grant No. 201707010151), and Dongguan Science Technology Commission (Grant No. 20185071511637). These organizations contribute to the promotion of this project, and they provide the technical support in the project.

Availability of data and material Data sharing not applicable to this article as no datasets were generated or analyzed during the current study. 


\section{Compliance with ethical standards}

Conflict of interest The authors declare that they have no conflict of interest.

\section{References}

1. Adler J (2010) Ceramic diesel particulate filters. Int J Appl Ceram Technol 2(6):429-439

2. Barrios CC, Martin C, Dominguez-Saez A et al (2014) Effects of the addition of oxygenated fuels as addictives on combustion characteristics and particle number and size distribution emissions of a TDI diesel engine. Fuel 132(1):93-100

3. Choi S, Oh KC, Lee CB (2014) The effects of filter porosity and flow conditions on soot deposition/oxidation and pressure drop in particulate filters. Energy 77:327-337

4. Djavareshkian MH, Esmaeli A, Parsani A (2011) Aerodynamics of smart flap under ground effect. Aerosp Sci Technol 15(8):642-652

5. Ji PS, Harrison RM, Brear F (1999) Particle size distribution from a modern heavy duty diesel engine. Sci Total Environ 235:305-317

6. Johnson TV (2010) Review of diesel emissions and control. Int J Engine Res 10(5):275-285

7. Ju H (2011) Study on formation laws and size distribution of soot in the diesel engines. Huazhong University of Science and Technology, Wuhan (in Chinese)
8. Liu X (2005) Emission and control of internal combustion engine. China Machine Press, Beijing (in Chinese)

9. Morawska L, Bofinger ND, Ladislav Kocis A et al (1998) Submicrometer and supermicrometer particles from diesel vehicle emissions. Environ Sci Technol 32(14):2033-2042

10. Tsuneyoshi K, Yamamoto K (2012) A study on the cell structure and the performance of wall-flow diesel particulate filter. Energy 48(1):492-499

11. Uystepruyst D, Krajnović S (2013) Numerical simulation of the transient aerodynamic phenomena induced by passing manoeuvres. J Wind Eng Ind Aerodyn 114:62-71

12. YiY (2006) Simulating the soot loading in wall-flow dpf using a three-dimensional macroscopic model//SAE 2006 World Congress \& Exhibition

13. Yuan Z (2013) Gas solid two phase flow and numerical simulation. Southeast University Press, Nanjing (in Chinese)

14. Zhang G (2006) Fluid mechanics. China Machine Press, Beijing (in Chinese)

15. Zhong $\mathrm{Y}$, Huang Q, Wu H (2017) Exhaust pollution and its control technology of vehicle diesel. Ind Technol 22:2033-3042 (in Chinese)

16. Zuccaro G, Lapenta G, Ferrero F et al (2011) Multiphase and multiphysics particle in cell simulation of soot deposition inside a diesel particulate filter single channel. Comput Phys Commun 182:347-359

Publisher's Note Springer Nature remains neutral with regard to jurisdictional claims in published maps and institutional affiliations. 